\title{
Quadrotor Control for RF Source Localization and Tracking
}

\author{
Jason T. Isaacs ${ }^{1}$, François Quitin ${ }^{2}$, Luis R. García Carrillo ${ }^{3}$, Upamanyu Madhow ${ }^{1}$, and João P. Hespanha ${ }^{1}$
}

\begin{abstract}
Preliminary results on quadrotor control strategies enabling omnidirectional radio frequency (RF) sensing for source localization and tracking are discussed. The use of a quadrotor for source localization and tracking requires a tight coupling of the attitude control and RF sensing designs. We present a controller for tracking a ramp reference input in yaw (causing rotation of quadrotor) while maintaining a constant altitude hover or translation. The ability to track a ramp in the yaw angle is crucial for RF bearing estimation using received signal strength (RSS) measurements from a directional antenna as it avoids the need for additional gimbaling payload. This bearing or angle of arrival (AOA) estimate is then utilized by a particle filter for source localization and tracking. We report on extensive experiments that suggest that this approach is appropriate even in complex indoor environments where multipath fading effects are difficult to model.
\end{abstract}

\section{INTRODUCTION}

We investigate the problem of $\mathrm{RF}$ source localization and tracking using a quadrotor equipped with a directional antenna. The RF beacon could originate from a source for search and rescue in civilian/military operations or a sensor that wishes to establish an on-demand high data rate link. The key hurdle in solving this problem is that, in the presence of reflectors, the RF signal strength does not vary monotonically with distance from the source. Consequently, source localization algorithms that rely on the gradient of the RSS can get stuck in local maxima. This problem can be solved by fitting the quadrotor with a directional antenna, rotating the quadrotor repeatedly, and using the RSS measurements as the robot rotates to infer the source direction. These direction estimates can then be combined with known positions of the quadrotor in a particle filter framework to estimate the location of the RF source.

We begin by mounting a directional antenna on a quadrotor and rotating the vehicle about its vertical axis, see Figure 1. As the antenna rotates, the dominant antenna lobe picks up the signal incident from different directions, providing us with the angular RSS pattern at that point in space. We pick the direction in which the RSS is maximum as our estimate of the bearing to the source. In the presence of reflectors and noise, this estimate is prone to have significant

*This work was supported by the Institute for Collaborative Biotechnologies through grant W911NF-09-0001 from the U.S. Army Research Office. The content of the information does not necessarily reflect the position or the policy of the Government, and no official endorsement should be inferred.

${ }^{1}$ J.T. Isaacs, U. Madhow, and J.P. Hespanha are with Department of Electrical and Computer Engineering, University of California, Santa Barbara $\{j t i s a a c s$, madhow, hespanha\}@ece.ucsb.edu

${ }^{2} \mathrm{~F}$. Quitin is with the School of Electrical and Electronic Engineering, Nanyang Technological University, fquitinentu.edu.sg

${ }^{3}$ L.R. García Carrillo is with the College of Science \& Engineering, Texas A\&M University - Corpus Christi,luis.garcia@tamucc. edu

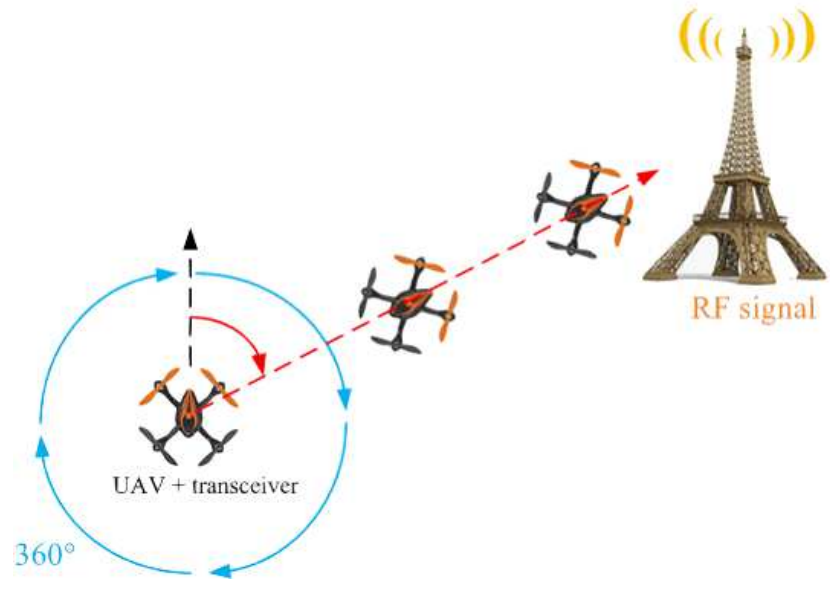

Fig. 1. The problem of RF source localization and tracking using a quadrotor equipped with a directional antenna.

errors. To allow for this possibility when performing the source localization, we use a particle filter with a noise model that assumes that some portion of measurements come from outliers.

Contributions: Our main contributions can be summarized as follows: 1) We develop an experimental platform consisting of a quadrotor and a directional antenna to localize and track an RF source. The proposed algorithm uses AOA estimates obtained by rotating the quadrotor as inputs to a particle filter used for localization and tracking. 2) We present a quadrotor control strategy that ensures continuous rotation about its vertical axis while maintaining a constant altitude hover or translation. This continuous rotation removes the need for a gimbaling mechanism for rotating the directional antenna for AOA estimation. 3) We report on experimental results that characterize the performance of the proposed algorithm in an indoor area. We find that even in the presence of outlier bearing measurements the particle filter is capable of localizing the RF source, thereby illustrating the efficacy of the algorithm.

Related Work: The fundamental problem of Direction of Arrival (DOA) estimation is considered in [1] and [2]. While [1] uses an actuated parabolic reflector to estimate the DOA, [2] infers the DOA by rotating the antenna around a signalblocking obstacle and choosing the direction in which the signal was most attenuated. In other related work, [3] uses a WMR equipped with an omnidirectional antenna to estimate the RSS gradient by measuring the power at many locations.

In [4] a rotating directional antenna is mounted on a wheeled mobile robot for the purpose of wireless node 
localization. The RSS is measured as a function of antenna angle and is cross-correlated with a known antenna gain pattern to determine the relative bearing between an unknown radio source and the mobile robot. A particle filter is used to determine the location of the stationary radio source. One major advantage of using autonomous vehicle for RF source localization is that the movement of the vehicle can be used to help with some of the estimation tasks such as sensor pointing. Unlike [4], where a dedicated servo-motor is used to rotate the antenna (also adding payload and power requirements), we propose a solution that fully exploits the mobility of the vehicle, and does not require additional hardware. A self-localization algorithm for a mobile robot team is proposed in [5]. Each robot is equipped with an antenna that provides a $\pi$ periodic estimate of the relative bearing between each pair of communicating agents. The proposed algorithm uses these bearing estimates in a MultiHypothesis Extended Kalman Filter (EKF) framework to localize all the agents. In [6], a mobile robot is equipped with a directional antenna for the purpose of localizing multiple stationary nodes in a wireless network by tracking the posterior distribution of the source location using a particle filter. Others have used particle filters for estimating the location of a source using bearing estimates including [7] and [8].

The control of quadrotor aircraft has attracted considerable attention from the academic community over the past decade. Many of the recent advances focus on aerobatic and aggressive maneuvers where the attitude deviates significantly from the hover state [9], [10], and [11]. However, in [12], it is pointed out that PID control is sufficient for attitude tracking in low velocity situations where the vehicle approximates double-integrator dynamics. While the controllers derived in this work are also intended for the near hover regime, tracking a ramp input in yaw during translation requires that we must account for the rotating reference frame in the roll and pitch controllers. In [13], the authors present a strategy based on imaging, inertial, and altitude sensors aiming at estimating and controlling the vehicle's heading orientation, relative position, and forward velocity, with respect to a specific trajectory that must be tracked. An on-board camera allows estimation of the vehicle heading angle with respect to the longitudinal orientation of the path. Similarly, the imaging sensor is used for stabilizing the lateral distance of the vehicle in order to navigate exactly over the trajectory. The performance of the proposed estimation and control strategies is tested in real-time experiments, validating the effectiveness of the proposed approach for accomplishing path following tasks.

\section{BEARING ESTIMATION}

In [4], a rotating antenna was used to determine the bearing to an RF source. It was shown that if an antenna with an appropriate radiation pattern is mounted onto a rotating device, the resulting RF signal will show a pattern that permits one to detect the bearing to the RF source. If the wireless propagation is dominated by the Line-of-Sight
(LoS) component, the RF power pattern for each rotation of the vehicle will be characterized by the antenna's radiation pattern. The bearing of the RF source can then be determined by matching the measured RF power pattern for one rotation with the antenna's radiation pattern [4]. In the case of very directional antennas, the yaw corresponding to the maximum RF power corresponds to the bearing to the source [14].

Of course, the presence of multipath will complicate this simple concept: each rotation will no longer exhibit the radiation pattern characteristics, but rather a weighted superposition of shifted radiation patterns, corresponding to each multipath. Another problem with this technique is that the RF source and the vehicle may be at different altitudes, and that the radiation pattern of the vehicle's antenna may change for different elevation angles. In that case, the RF power pattern for each rotation may vary depending on whether the vehicle is close or far away from the source.

\section{QuAdrotor DynAmicAl MODEL AND CONTROL}

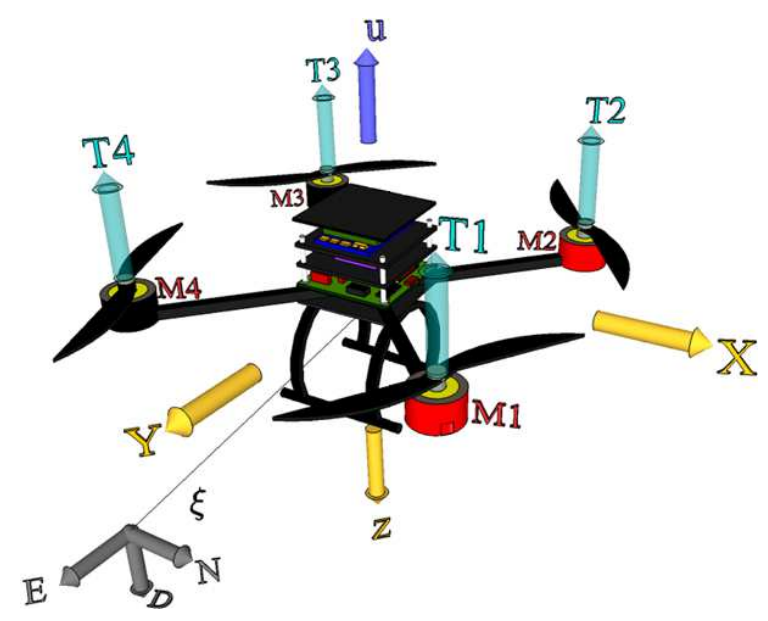

Fig. 2. NED diagram of the rotorcraft dynamical model.

In the following, we start by introducing the dynamical model used for the controller derivation. We then derive the controllers for constant hovering while rotating and continuous translation while rotating. Finally, we show how these control strategies can be implemented on the AR.Drone. This section presents the dynamic model equations for an "X-type" quadrotor configuration. The vehicle's dynamical model considers six degrees of freedom and is presented on Newton-Euler formalism, with dynamics expressed in NorthEast-Down (NED) inertial and body-fixed coordinates, see Figure 2.

\section{A. Dynamical Model}

Let $\mathcal{I}=\{N, E, D\}$ and $\mathcal{B}=\{X, Y, Z\}$ represent an inertial reference frame and a body-fixed frame, respectively. The position vector of the center of mass of the rotorcraft relative to the inertial frame is denoted by $\xi=(x, y, z)$, while the orientation vector (attitude) with respect to (w.r.t.) the inertial frame is expressed by $\eta=(\psi, \theta, \phi)$. The terms $\psi, \theta$, and $\phi$ are denoted as yaw, pitch, and roll Euler angles, 
respectively. The full nonlinear dynamics of the quad-rotor can be expressed as

$$
\begin{aligned}
m \ddot{\xi} & =-m g D+\mathcal{R} F \\
\mathbb{I} \dot{\Omega} & =-\Omega \times \mathbb{I} \Omega+\tau
\end{aligned}
$$

where $\mathcal{R} \in S O(3)$ is a rotation matrix that associates the inertial frame with the body-fixed frame, $F$ denotes the total force applied to the vehicle, $m$ is the rotorcraft mass, $g$ denotes the gravitational constant, $\Omega$ represents the angular velocity of the vehicle expressed in the body fixed frame, I describes the inertia matrix, and $\tau$ is the total torque.

A simplified mathematical model for the quad rotorcraft that does not take into account all nonlinearities, but simplifies the development of the control strategy and serves well for capturing the essence of the vehicle's dynamics can be expressed as [15]:

$$
\begin{aligned}
m \ddot{x} & =-u(\cos (\psi) \sin (\theta) \cos (\phi)+\sin (\psi) \sin (\phi)) \\
m \ddot{y} & =-u(\sin (\psi) \sin (\theta) \cos (\phi)-\cos (\psi) \sin (\phi)) \\
m \ddot{z} & =-u(\cos (\theta) \cos (\phi))+m g \\
\ddot{\psi} & =\tilde{\tau}_{\psi} \\
\ddot{\theta} & =\tilde{\tau}_{\theta} \\
\ddot{\phi} & =\tilde{\tau}_{\phi}
\end{aligned}
$$

where $m$ is the rotorcraft mass, and $g$ denotes the gravitational constant.

\section{B. Control Strategy}

This subsection presents a control strategy for the quad rotorcraft that ensures continuous rotation about the Z-axis while maintaining a hover flight at constant altitude, as well as a continuous rotation about the $Z$-axis while performing translational displacements in the $N-E$ plane.

1) Altitude control: The altitude dynamic $z$ of the rotorcraft is stabilized by using the following control input in equation (5):

$$
u=m\left(-k_{d z} \dot{z}-k_{p z} e_{z}+g\right) \frac{1}{\cos \theta \cos \phi}
$$

with $e_{z}=z_{d}-z$ as the $z$ error position and $z_{d}$ as the rotorcraft desired altitude. The terms $k_{d z}$ and $k_{p z}$ are positive constants of a PD controller which should be carefully chosen to ensure a stable well-damped response in the vertical axis.

2) Yaw control: The following control input is applied in equation (6) for stabilizing the heading dynamic $\psi$

$$
\tilde{\tau}_{\psi}=k_{p \psi}\left(\psi_{d}-\psi\right)-k_{d \psi} \dot{\psi}+k_{i \psi} \int_{0}^{t}\left(\psi_{d}-\psi\right) d \tau
$$

where $\psi_{d}$ represents the desired heading angle. The terms $k_{p \psi}, k_{i \psi}$, and $k_{d \psi}$ denote the positive constants of a PID controller.
Introducing equations (9) and (10) into (3)-(6), and provided that $\cos \theta \cos \phi \neq 0$, one has

$$
\begin{aligned}
m \ddot{x} & =-m g\left(\cos (\psi) \tan (\theta)+\frac{\sin (\psi) \tan (\phi)}{\cos (\theta)}\right) \\
m \ddot{y} & =-m g\left(\sin (\psi) \tan (\theta)-\frac{\cos (\psi) \tan (\phi)}{\cos (\theta)}\right) \\
m \ddot{z} & =-k_{d z} \dot{z}-k_{p z} e_{z} \\
\ddot{\psi} & =k_{p \psi}\left(\psi_{d}-\psi\right)-k_{d \psi} \dot{\psi}+k_{i \psi} \int_{0}^{t}\left(\psi_{d}-\psi\right) d \tau
\end{aligned}
$$

The vehicle is considered to operate only in regions where $-\pi / 2<\theta<\pi / 2$ and $-\pi / 2<\phi<\pi / 2$, which ensures that the trajectory does not pass through any singularities [16].

From equation (13) and (14) it follows that $z \rightarrow z_{d}$ and $\psi \rightarrow \psi_{d}$. Therefore, once the heading of the vehicle coincides with the desired heading, displacement in the longitudinal and lateral directions can be obtained from the following equations

$$
\begin{aligned}
{ }^{\mathcal{B}} \ddot{x} & =-g \tan \theta \\
{ }^{\mathcal{B}} \ddot{y} & =g \frac{\tan \phi}{\cos \theta}
\end{aligned}
$$

where ${ }^{\mathcal{B}} \ddot{x}$ and ${ }^{\mathcal{B}} \ddot{y}$ represent parameters expressed with respect to the body-fixed frame.

The following control strategies are based on the idea that the overall model expressed with equations (3)-(8), comprises two subsystems, i.e., the attitude dynamics system and the position dynamics system, with a time-scale separation between them. From this perspective, it is possible to propose a hierarchical control scheme, where the $x$ and $y$ positioning controller provides the reference attitude angles $\theta_{d}, \phi_{d}$, respectively, which are the angles that must be tracked by the low level attitude controllers.

3) Forward position and pitch angle control: Consider the subsystem given by equations (7) and (15). To further simplify the analysis, let's impose a very small upper bound on $|\theta|$ in such a way that the difference $\tan (\theta)-\theta$ is arbitrarily small $(\theta \approx \tan (\theta))$. Therefore, the subsystem (7) and (15) is reduced as

$$
\begin{aligned}
{ }^{\mathcal{B}} \ddot{x} & =-g \theta \\
\ddot{\theta} & =\tilde{\tau}_{\theta}
\end{aligned}
$$

To stabilize the pitch angular position (18), one can apply

$$
\tilde{\tau}_{\theta}=k_{p \theta}\left(\theta_{d}-\theta\right)-k_{d \theta} \dot{\theta}
$$

where $k_{p \theta}$ and $k_{d \theta}$ represent the positive constants of a PD controller. Finally, in order to stabilize the vehicle at a desired position $x_{d}$ the following virtual controller is defined

$$
\tilde{\theta}_{d}=k_{p x}\left(x_{d}-x\right)+k_{d x}\left(\dot{x}_{d}-\dot{x}\right)
$$

The controller gains $k_{p x}$ and $k_{d x}$ are chosen to ensure that $s^{2}+k_{d x} s+k_{p x}$ is a Hurwitz stable polynomial. The pitch reference angle $\tilde{\theta}_{d}$ represents the angle that must be tracked by the low level attitude controller. 
4) Lateral position and roll angle control: Consider the subsystem given by equations (8) and (16). Imposing a very small upper bound on $|\phi|$ in such a way that the difference $\tan (\phi)-\phi$ is arbitrarily small, i.e., $\phi \approx \tan (\phi)$, the subsystem given by equations (8) and (16) reduces to

$$
\begin{aligned}
{ }^{\mathcal{B}} \ddot{y} & =g \phi \\
\ddot{\phi} & =\tilde{\tau}_{\phi}
\end{aligned}
$$

which represents four integrators in cascade. To stabilize the roll angular position (22), one can apply

$$
\tilde{\tau}_{\phi}=k_{p \phi}\left(\phi_{d}-\phi\right)-k_{d \phi} \dot{\phi}
$$

where $k_{p \phi}$ and $k_{d \phi}$ represent the positive constants of a PD controller. We define a controller for the roll angle control in a similar manner to (17) - (20), resulting in the following virtual controller

$$
\tilde{\phi}_{d}=k_{p y}\left(y_{d}-y\right)+k_{d y}\left(\dot{y}_{d}-\dot{y}\right)
$$

where $k_{p y}$ and $k_{d y}$ are stabilizing control gains. The roll reference angle $\tilde{\phi}_{d}$ represents the angle that must be tracked by the low level attitude controller.

\section{Controller for Continuous Rotation and Translation}

As previously mentioned, two behaviors are required in the present application: rotation about the $Z$-axis while maintaining a hover flight at constant altitude, and rotation about the $Z$-axis while performing translational displacements in the $N-E$ plane at constant altitude. For both scenarios, the continuous rotation about the $Z$-axis is achieved by choosing a desired heading angle $\psi_{d}$ in equation (10) in the form of a ramp signal.

On one hand, when the control strategy is designed for a hover flight at constant altitude, the position coordinates where the hover flight will be performed are chosen as $\left(x_{d}, y_{d}\right)=\left(x_{0}, x_{0}\right)$, with $x_{0}$ and $y_{0}$ representing the rotorcraft initial position in the $N-E$ plane. In the other hand, when the vehicle is required to perform a translational displacement, the continuous rotation around the $Z$-axis needs to be taken into account in the computation of the desired direction of displacement. This is achieved by applying a rotation about the $Z$-axis to the reference angles $\left(\tilde{\theta}_{d}, \tilde{\phi}_{d}\right)$ as follows

$$
\left[\begin{array}{l}
\theta_{d} \\
\phi_{d}
\end{array}\right]=\left[\begin{array}{cc}
\cos \psi & -\sin \psi \\
\sin \psi & \cos \psi
\end{array}\right]\left[\begin{array}{c}
\tilde{\theta}_{d} \\
\tilde{\phi}_{d}
\end{array}\right]
$$

This procedure ensures that the translational displacement of the vehicle is performed towards the desired $\left(x_{d}, y_{d}\right)$ position, even when the vehicle is undergoing a constant rotation.

\section{Implementation of the Control Strategy for the AR.Drone}

The platform chosen for running the experimental applications consists of an AR.Drone from Parrot. The airframe of the AR.Drone is built from plastic and carbon fiber parts and measures $0.57 \mathrm{~m}$ across. The AR.Drone has a miniaturized inertial measurement unit (IMU) which allows measuring the pitch, roll, and yaw vehcile's dynamics for use in attitude stabilization. The onboard sensors include an ultrasonic rangefinder which is used to measure the vehicle's altitude up to $6 \mathrm{~m}$. The rotors are powered by 15 watts brushless motors powered by an 11.1 Volt lithium polymer battery. This source provides approximately 12 minutes of operation, when the vehicle is flown at speeds of around $5 \mathrm{~m} / \mathrm{s}$.

The onboard computer runs a Linux operating system and communicates with a supervisory ground station through a Wi-Fi hotspot. The built-in autopilot of the AR.Drone takes as input four parameters: a reference angle for the pitch dynamic, a reference angle for the roll dynamic, a desired velocity for the altitude dynamic, and a desired velocity for the heading dynamic. Specifically, the desired pitch and roll angles are generated in the supervisory ground station using the formula shown in equation (25). Once received by the autopilot, the onboard controller is able to compute the necessary control inputs $\tilde{\tau}_{\theta}$ and $\tilde{\tau}_{\phi}$. This process generates the required translational displacement along the $N$ and $E$ axes, respectively.

In order to fulfill the specifications of the AR.Drone concerning the control signals for $z$ and $\psi$ dynamics, we designed slightly modified versions of equations (9) and (10), which are computed in the supervisory ground station. Specifically, the reference signal for the vertical velocity is obtained as follows

$$
\dot{z}_{d}=-k_{p z}\left(z_{d}-z\right)-k_{d z} \dot{z}
$$

with $z, \dot{z}$ coming from the motion capture system, and gains $k_{p z}$ and $k_{d z}$ chosen to ensure that $s^{2}+k_{d z} s+k_{p z}$ is a Hurwitz stable polynomial. Similarly, the reference signal for the heading velocity is computed as

$$
\dot{\psi}_{d}=k_{p \psi}\left(\psi_{d}-\psi\right)-k_{d \psi} \dot{\psi}+k_{i \psi} \int_{0}^{t}\left(\psi_{d}-\psi\right) d \tau
$$

with with $\psi, \dot{\psi}$ obtained from the motion capture system, and terms $k_{p \psi}, k_{i \psi}$, and $k_{d \psi}$ denote the positive constants of a PID controller. Block diagrams describing the the implementation of the controllers in equations (26) and (27) are shown in Figure 3 and 4, respectively.

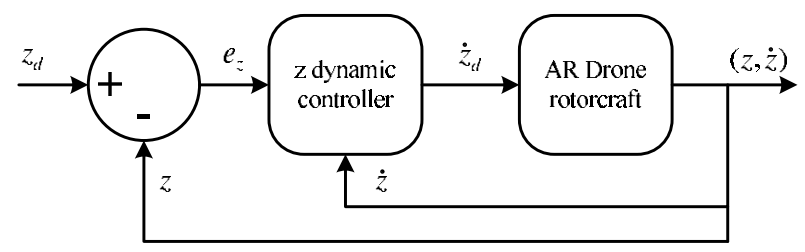

Fig. 3. Block diagram of the controller for stabilizing the AR.Drone altitude.

\section{PARTicle Filter}

In this section, we describe the use of a multinomial resampling particle filter [17] for estimating the location of a source using bearing-only estimates. This type of particle filter was chosen as it is well suited for the measurement 


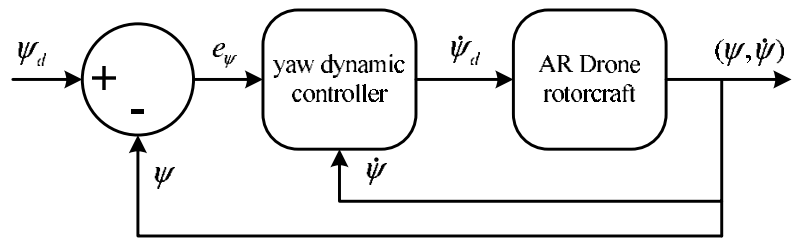

Fig. 4. Block diagram of the controller for stabilizing the AR.Drone heading.

noise model described in Section V-B. The state-space model of the RF source is given by

$$
p_{k}=F p_{k-1}+v_{k}
$$

where $p_{k}=\left[p_{x, k}, p_{y, k}, \dot{p}_{x, k}, \dot{p}_{y, k}\right]^{T}$ is the state vector containing the $\mathrm{x}$ - and $\mathrm{y}$-coordinate of the source at cycle $k$, and the $\mathrm{x}$ - and $\mathrm{y}$-speed of the source at cycle $k$, respectively, and $v_{k}$ is a noise term, modeled with a zero-mean Gaussian distribution. The $4 \times 4$ transition matrix $F$ is defined by

$$
F=\left[\begin{array}{llll}
1 & 0 & d T & 0 \\
0 & 1 & 0 & d T \\
0 & 0 & 1 & 0 \\
0 & 0 & 0 & 1
\end{array}\right]
$$

where $d T$ is the time interval between two measurements. The measurement model, based on a bearing-only measurement, is given by

$$
\zeta_{k}=h\left(p_{k}, \xi_{k}\right)+w_{k}
$$

where $h(p, \xi)=\operatorname{atan} 2\left(p_{y}-y, p_{x}-x\right)($ with $\operatorname{atan} 2(y, x)$ being the four quadrant inverse tangent function), $p_{k}$ is the state vector of the source at cycle $k$ and $\xi_{k}$ is the vector containing the coordinates of the quadrotor at cycle $k$. The term $w_{k}$ is the measurement noise, that can be modeled as described in Section V-B to account for both measurement noise and outlier measurements. The particle filtering can be divided into two stages: one update stage and one resampling stage. During the update stage, each particle $l$ is first propagated with the state-space model:

$$
p_{k}^{(l)}=F p_{k-1}^{(l)}+v_{k}^{(l)}
$$

The error between the bearing measurement $\zeta_{k}$ and the bearing between quadrotor and the $l$-th particle $\zeta_{k}^{(l)}$ is given by $\varepsilon_{k}^{(l)}$, and is defined as follows:

$$
\begin{aligned}
& \zeta_{k}^{(l)}=h\left(p_{k}^{(l)}, \xi_{k}\right) \\
& \varepsilon_{k}^{(l)}=\zeta_{k}-\zeta_{k}^{(l)}
\end{aligned}
$$

where the last subtraction is wrapped to $[-\pi, \pi)$. Finally, each particle is given a weight $q_{k}^{(l)}$ as follows:

$$
q_{k}^{(l)}=f_{\varepsilon}\left(\varepsilon_{k}^{(l)}\right)
$$

where $f_{\varepsilon}$ is the probability density function of the measurement error (containing both measurement error and outliers, as defined in Section V-B). The weights are then normalized so that $\sum_{l=1}^{L} q_{k}^{(l)}=1$ (where $L$ is the total number of particles).

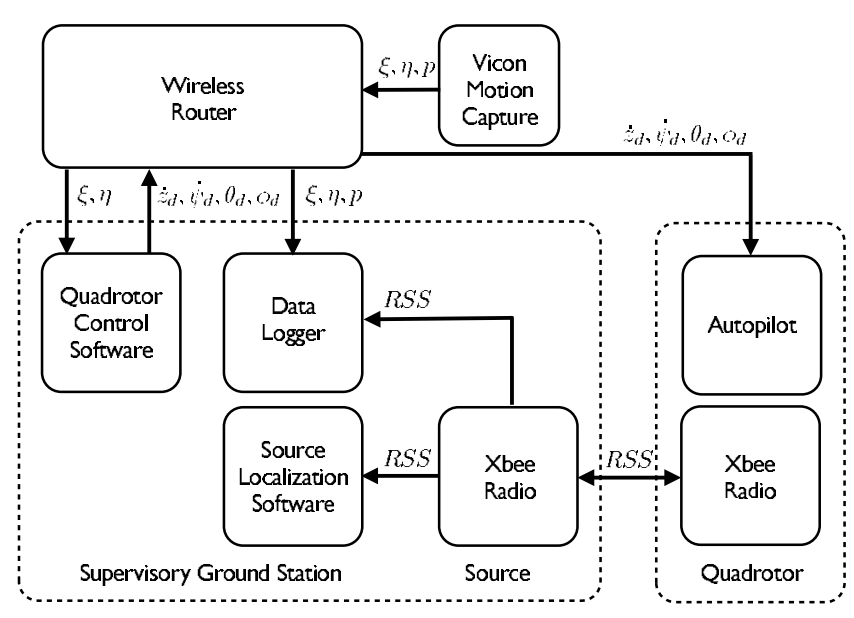

Fig. 5. The experimental testbed architecture is shown with arrows representing information exchange between modules.

In practice, particles with bearing to the source $\zeta_{k}^{(l)}$ that differ a lot from the bearing measurement $\zeta_{k}$ will end up having lower weights, and will have a higher probability of getting discarded during the resample stage. In the resample stage, the particles with lowest probability are discarded, and are resampled as particles with high probability. In this paper, we use the Multinomial Resampling algorithm to resample the particles. The idea behind multinomial resampling is that, after resampling, the number of occurrences of each state (given by the number of particles in that state) will correspond to the estimated multinomial distribution of the state [17].

\section{EXPERIMENTAL RESULTS}

\section{A. Experimental testbed}

The experimental testbed architecture is shown in Figure 5. In this setup, the RF source (Xbee radio) is collocated with the ground control station as would be the case in a cooperative scenario where the quadrotor is used to provide localization and navigation support to a ground agent. A second Xbee radio is mounted on an AR.Drone quadrotor and is configured for remote control. The Xbee radio located at the ground station is equipped with an omni-directional antenna and sends a message to the Xbee located on the quadrotor requesting the most recent RSS measurement. A Vicon Motion Capture system is used to measure ground truth values for the quad rotor $(\xi$ and $\eta$ ) as well as the RF source position, $p$. A photograph of the AR.Drone test platform showing the mounting of the Xbee tranceiver and directional antenna can be seen in Figure 6.

\section{B. RF source bearing estimate}

The antenna used in our setup is WA5VJB Quad-patch antenna. This antenna has a half-power beamwidth of approximately $40^{\circ}$, and the front-to-back ratio of the antenna is in the order of $30 \mathrm{~dB}$. Figure 7 shows the received signal strength (RSS) when the quadrotor is rotating at a fixed position with respect to the source. A regular pattern can be observed: the peaks correspond to when the main 


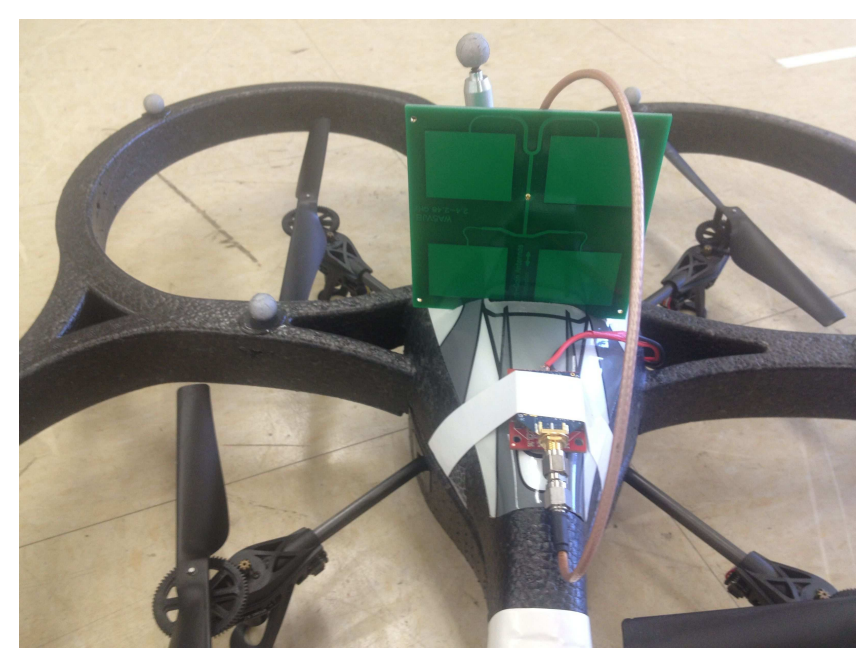

Fig. 6. The AR.Drone experimental test platform is shown with an Xbee transceiver and a directional antenna.

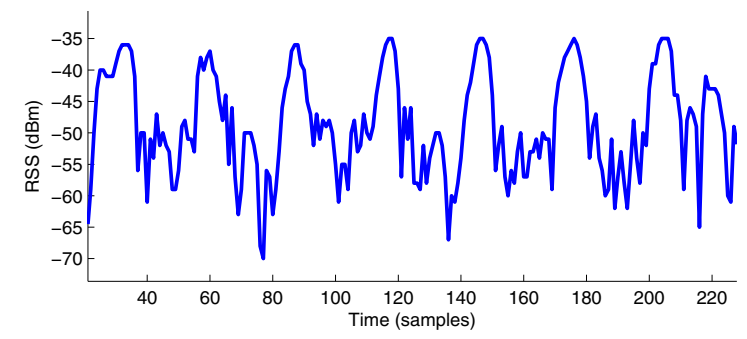

Fig. 7. RSS when the quadrotor is rotating in a fixed position. A regular pattern can be observed, which correspond to the radiation pattern for each rotation.

beam of the antenna is directed towards the source. In that case, the LoS falls within the main beam of the antenna and dominates the RSS. When the antenna is turned away from the source, the LoS is strongly attenuated by the radiation pattern, resulting in lower RSS. By using the yaw corresponding to the maximum RSS over each rotation, the bearing of the RF source can be determined. Figure 8 shows the source bearing estimates for different quadrotor positions. In this test, the quadrotor was performing several rotations at four different locations around the source. The bearing estimates are consistent with the real source bearing, although some outliers can be observed when the quadrotor is very close to the source. This could be due to lack of directionality in the antenna gain pattern when the source is directly under the quadrotor. By running several similar tests, the error of our bearing estimation scheme could be determined experimentally. Similarly to [14], the probability density function of the bearing error is characterized as

$$
f_{\varepsilon}(\varepsilon)=\left(1-p_{o}\right) X+p_{o} U_{2 \pi}
$$

where $X \sim\left(\mu, \sigma^{2}\right)$ is a zero-mean Gaussian distributed random variable, $U_{2 \pi}$ is an uniform random variable distributed over $[0,2 \pi)$, and $p_{o}$ is the probability of measuring an outlier. The first term in equation (30) represents the angle estimation error, while the second term corresponds to the probability

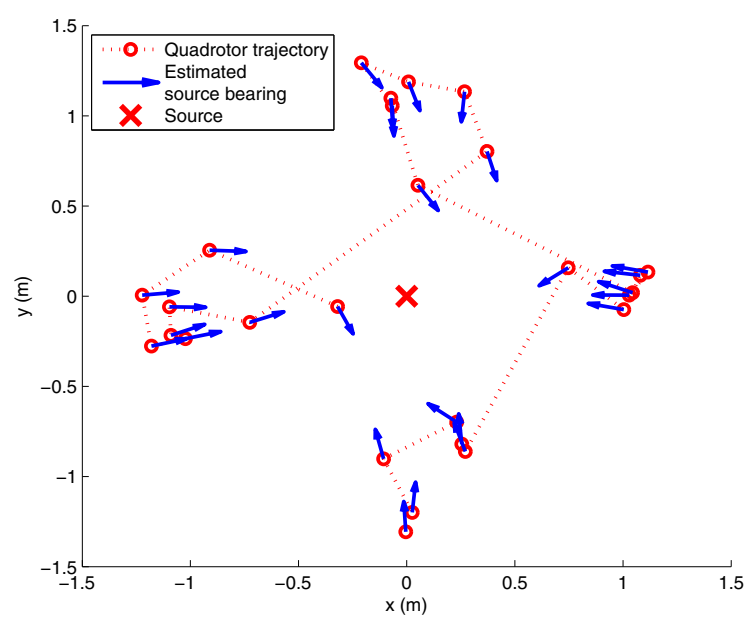

Fig. 8. Source bearing estimates for different quadrotor positions.

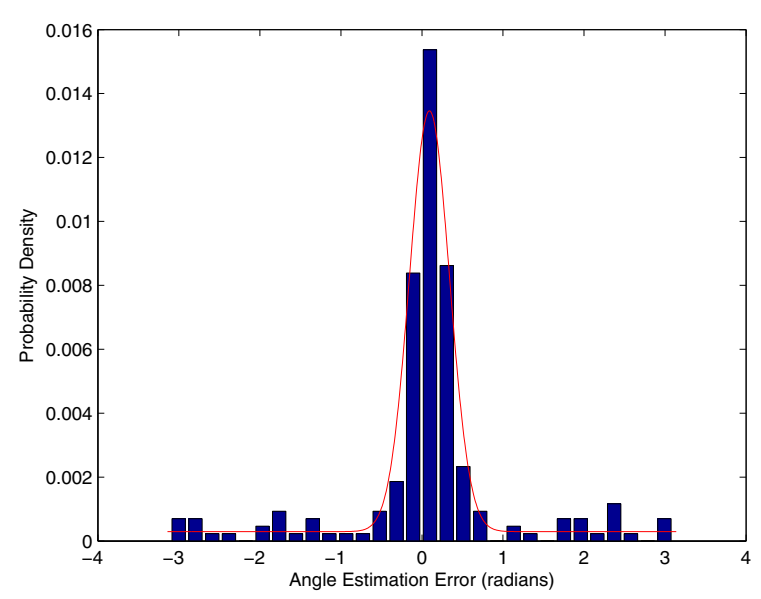

Fig. 9. Source bearing estimation errors.

of an outlier measurement (e.g. caused by a strong reflected multipath). The numerical values obtained from our experiment are $p_{o}=0.19, \mu=0.0929 \mathrm{rad}$, and $\sigma=0.2468 \mathrm{rad}$. The normalized histogram of bearing estimation errors and the resulting probability density function (30) can be seen in Figure 9.

\section{Quadrotor control}

Several experiments were conducted to tune and verify each of the quadrotor control loops (20), (24), (26), and (27). We found the altitude (26) and yaw (27) controllers to be straightforward to tune for the hover while rotating case. However, when the additional translational controllers (20) and (24) were introduced the ability of the yaw controller to track a ramp suffered. This is possibly due to the limited overall torque budget that is shared among all the controllers. It appeared as though the internal AR.drone controllers placed a higher priority on the translational controllers if the torque limit was reached. To work around this limitation we put a limit on the maximum desired roll and pitch angles. 

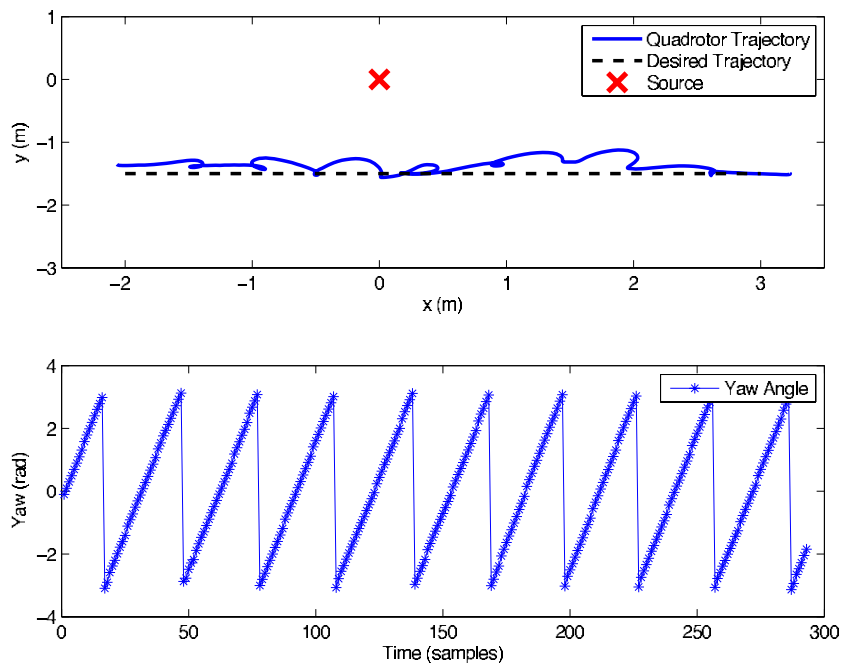

Fig. 10. The top panel shows the measured position of the quadrotor (blue), the desired trajectory (black dashed), and the source position (red $\mathrm{x}$ ) during an experiment. The bottom panel shows the measured yaw of the quadrotor during the corresponding test.

This allowed enough control authority to successfully track the ramp input, but resulted in a limited translational speed. As can be seen in the example trajectory from Figure 10, the measured yaw is increasing at a constant rate (bottom panel) while the quadrotor is translating along the desired trajectory (top panel).

\section{Source localization}

The particle filter described in Section IV was used to determine the location of the RF source, based on the bearing estimates only. In a first series of tests, the source was static and located at the center of the testing region. The quadrotor was flying to four navigation points located all around the source repeatedly. Figure 11 shows the particle filter at several stages of the test. Initially (subfigure (a)), the particles are spread out randomly over the whole testing region. After a few iterations (subfigure (b)), several angle estimates have been obtained. The particles then concentrate in a cone from the quadrotor to the direction of the source. Because the quadrotor has only had one (angular) view of the source, it is unable to resolve the exact location of the source. Once the quadrotor moves to another location (subfigure (c)), it gets a new (angular) view of the source, and the particles start to collapse to the source's location. Finally, once the quadrotor has had many views of the source (subfigure (d)), all particles have converged to the source's true location. A snapshot of this experiment is shown in Figure 13.

Another series of tests were conducted, where the source was located on one side of the testing region and the quadrotor was flying back and forth along a line at the other side of the testing region. The results, shown in Figure 12 are roughly the same as previously: as soon as the quadrotor has had different views of the source, all particles start to converge to the source's true location. Note that the filter is not able to resolve the location of the source along the $y$-axis
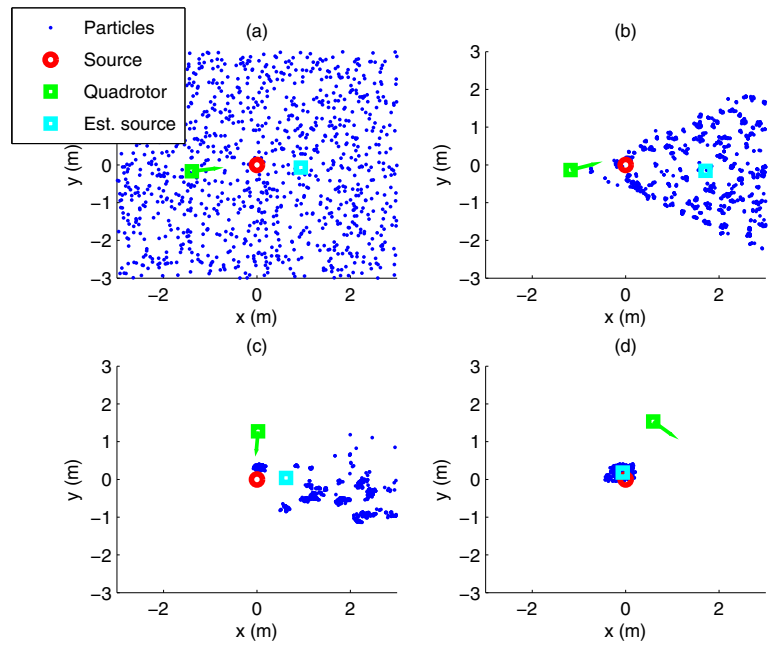

Fig. 11. Particle filter for a static source, and the quadrotor flying around the source. Once the quadrotor has had multiple views of the source, the particles start collapsing to the source's location.
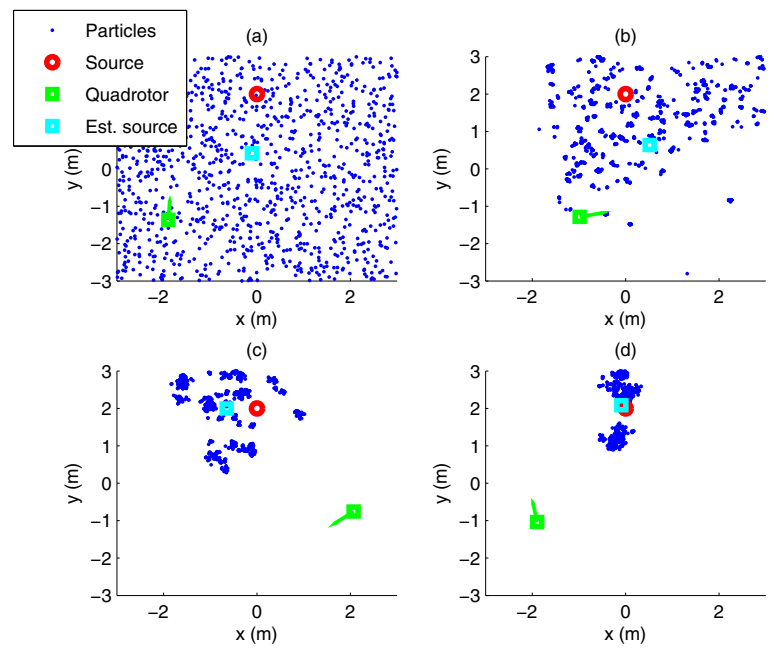

Fig. 12. Particle filter for a static source, and the quadrotor flying along a line opposite to the source. The particles converge to the source's location after the quadrotor has had multiple views of the source, but an uncertainty remains among the $y$-axis due to the quadrotor's trajectory.

completely, as the quadrotor never had a clear view of that axis.

In Figure 14, the source is moving in a circle, and the quadrotor is flying to four navigation points located around the source region. After some iterations the particles collapse to the source's true location. As the source is moving, the quadrotor keeps estimating the bearing to the source, and the particle filter is able to track the source's true location. Note that if the movement of the source is too fast with respect to the quadrotor's rotating speed, the update rate of the particle filter will be too slow to track the source's location. A snapshot of the moving source experiment can be seen in Figure 15. A video of the RF source localization and tracking experiments can be seen at http://www.youtube.com/watch?v=FiJ1N52XeXY 


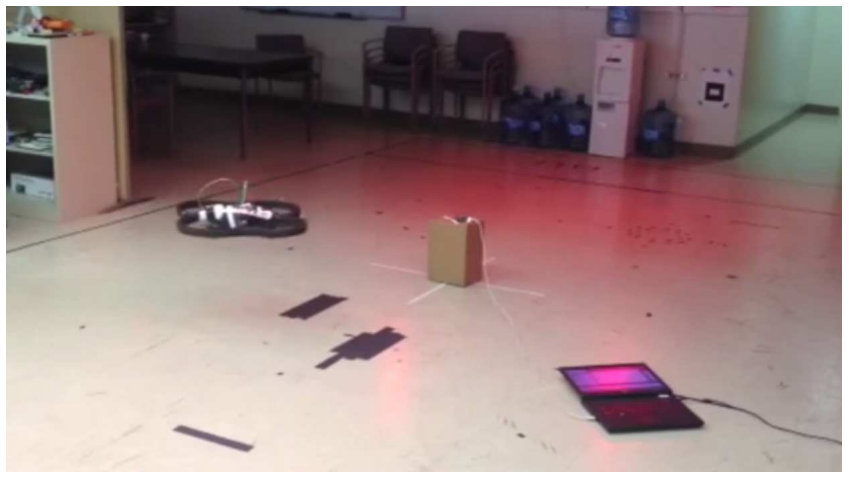

Fig. 13. Real time experimental application for the case of a static source. The quadrotor can be seen flying around the source.

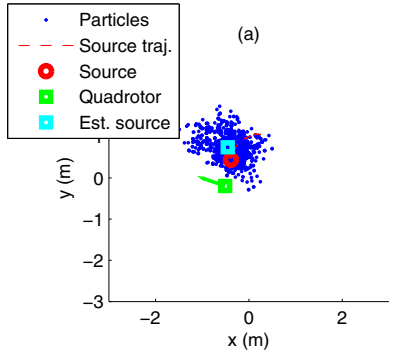

(c)
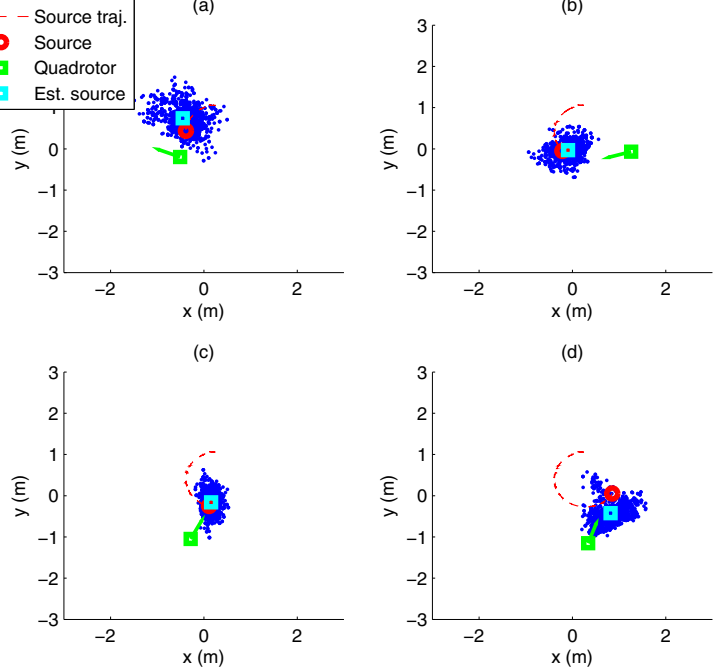

(d)

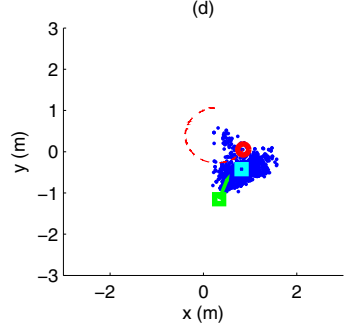

Fig. 14. Particle filter for a moving source, and the quadrotor flying around the source. If the quadrotor is able to get multiple views of the source while the source is moving, the particle cloud is able to track the source's position.

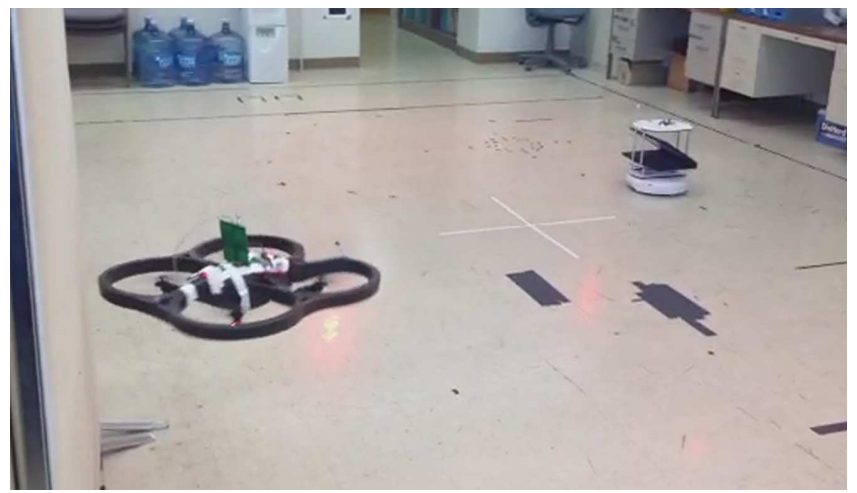

Fig. 15. Real time experimental application for the case of a moving source. The source is moving in a circle, and the quadrotor is flying to four navigation points located around the source region.

\section{CONCLUSIONS}

We have proposed and demonstrated a method for RF source localization and tracking using a quadrotor and a directional antenna. The key idea, is to exploit the mobility of the quadrotor and derive bearing estimates by rotating the body of the aircraft (and thus the rigidly mounted antenna). A particle filter is shown to work well in practice for both source localization and tracking of a mobile source provided the quadrotor can position itself to allow for diverse sensing angles. Indoor experiments demonstrate the efficacy of this approach even when the bearing measurements contain outliers due to strong reflected multipath signals.

A topic of current work involves adapting this approach for outdoor use. Ultimately, the algorithms running on the supervisory ground station would be embedded on the quadrotor controller. While we expect an improvement in the performance of the bearing estimation in an open area devoid of significant reflectors, uncertainty in the quadrotor self-localization (GPS or dead reckoning) will impact the performance of the particle filter. Additional filters will also be necessary to estimate the states of the quadrotor given that onboard sensors will replace the Vicon motion capture system. Another direction of interest involves using a single quadrotor to aid in the localization of several ground agents. In this scenario, quadrotor path planning is a critical design element for maintaining localization accuracy.

\section{REFERENCES}

[1] B. Hood and P. Barooah, "Estimating DoA from radio-frequency RSSI measurements using an actuated reflector," IEEE Sensors Journal, vol. 11, no. 2, pp. 413-417, 2011.

[2] Z. Zhang, X. Zhou, W. Zhang, Y. Zhang, G. Wang, B. Y. Zhao, and H. Zheng, "I am the antenna: accurate outdoor AP location using smartphones," in Conference on Mobile Computing and Networking, Las Vegas, NV, 2011, pp. 109-120.

[3] K. Dantu, P. Goyal, and G. S. Sukhatme, "Relative bearing estimation from commodity radios," in IEEE International Conference on Robotics and Automation, Kobe, Japan, May 2009, pp. 3215-3221.

[4] J. Graefenstein, A. Albert, P. Biber, and A. Schilling, "Wireless node localization based on RSSI using a rotating antenna on a mobile robot," in Workshop on Positioning, Navigation and Communication, Hanover, Germany, March 2009, pp. 253-259.

[5] J. Derenick, J. Fink, and V. Kumar, "Localization using ambiguous bearings from radio signal strength," in International Conference on Intelligent Robots and Systems, San Francisco, CA, Sept 2011.

[6] D. Song, J. Yi, and Z. Goodwin, "Localization of unknown networked radio sources using a mobile robot with a directional antenna," in American Control Conference, New York, NY, July 2007.

[7] A. Mannesson, M. Yaqoob, F. Tufvesson, and B. Bernhardsson, "Radio and IMU based indoor positioning and tracking," in Systems, Signals and Image Processing (IWSSIP), 2012 19th International Conference on, 2012, pp. 32-35.

[8] A. Tilton, T. Yang, H. Yin, and P. Mehta, "Feedback particle filterbased multiple target tracking using bearing-only measurements," in Information Fusion (FUSION), 2012 15th International Conference on, 2012, pp. 2058-2064.

[9] D. Mellinger and V. Kumar, "Minimum snap trajectory generation and control for quadrotors," in Proceedings of the International Conference on Robotics and Automation. IEEE, 2011, pp. 2520-2525.

[10] M. Hehn and R. DAndrea, "Quadrocopter trajectory generation and control," in Proceedings of the IFAC World Congress, vol. 18, no. 1, 2011, pp. 1485-1491.

[11] M. Cutler and J. P. How, "Actuator constrained trajectory generation and control for variable-pitch quadrotors," in Proceedings of the AIAA Guidance, Navigation, and Control Conference, 2012. 
[12] G. M. Hoffmann, H. Huang, S. L. Waslander, and C. J. Tomlin, "Quadrotor helicopter flight dynamics and control: Theory and experiment," in Proceedings of the AIAA Guidance, Navigation, and Control Conference, vol. 2, 2007.

[13] L. García Carrillo, G. Flores Colunga, G. Sanahuja, and R. Lozano, "Quad rotorcraft switching control: An application for the task of path following," IEEE Transactions on Control Systems Technology, vol. PP, no. 99, pp. 1-1, 2013.

[14] S. Venkateswaran, J. T. Isaacs, K. Fregene, R. Ratmansky, B. M. Sadler, J. P. Hespanha, and U. Madhow, "RF Source-Seeking by a Micro Aerial Vehicle using Rotation-Based Angle of Arrival Estimates," in American Control Conference, 2013.

[15] L. R. García Carrillo, A. E. Dzul López, R. Lozano, and C. Pégard, Quad Rotorcraft Control: Vision-Based Hovering and Navigation. Springer Publishing Company, Incorporated, 2012.

[16] T. Koo and S. Sastry, "Output tracking control design of a helicopter, model based on approximate linearization," in IEEE Conference on Decision and Control, Tampa, FL, USA, 1998.

[17] J. Hol, T. Schon, and F. Gustafsson, "On resampling algorithms for particle filters," in Nonlinear Statistical Signal Processing Workshop, 2006 IEEE, 2006, pp. 79-82. 